\title{
Entre modèle et rituel. Stratégies de contrôle du lien social dans la société urbaine idéale du Volgarizzamento del libro de' costumi e degli offizii de' nobili sopra il giuoco degli scacchi ( $\mathrm{XIV}^{\mathrm{e}}$ siècle)
}

Tra modello e rituale. Strategie di controllo dei legami sociali nella società urbana del Volgarizzamento del libro de' costumi e degli offizii de' nobili sopra il giuoco degli scacchi (XIV secolo).

\section{Alessandra Stazzone}

\section{OpenEdition}

\section{Journals}

Édition électronique

URL : http://journals.openedition.org/cei/1012

DOI : 10.4000/cei.1012

ISSN : 2260-779X

\section{Éditeur}

UGA Éditions/Université Grenoble Alpes

\section{Édition imprimée}

Date de publication : 31 octobre 2012

Pagination : 17-36

ISBN : 978-2-84310-234-9

ISSN : 1770-9571

Référence électronique

Alessandra Stazzone, «Entre modèle et rituel. Stratégies de contrôle du lien social dans la société urbaine idéale du Volgarizzamento del libro de' costumi e degli offizii de' nobili sopra il giuoco degli scacchi (xiv e siècle) », Cahiers d'études italiennes [En ligne], 15 | 2012, mis en ligne le 30 avril 2014, consulté le 27 mars 2021. URL : http://journals.openedition.org/cei/1012 ; DOI : https://doi.org/10.4000/cei.1012 


\title{
ENTRE MODÈLE ET RITUEL. STRATÉGIES DE CONTRÔLE DU LIEN SOCIAL DANS \\ LA SOCIÉTÉ URBAINE IDÉALE DU VOLGARIZZAMENTO \\ DEL LIBRO DE' COSTUMI E DEGLI OFFIZII DE' NOBILI SOPRA IL GIUOCO DEGLI SCACCHI (XIV ${ }^{\mathrm{e}}$ SIÈCLE)
}

\author{
Alessandra Stazzone \\ Université Paris - Sorbonne Paris 4
}

Chess is like life. Whether the game and pieces represent war, court society, or the world at large, in a general way and simply seen, the game has come to symbolize the world and human activity. This metaphor comes down from the thirteenth century when Jacob de Cessolis, a Dominican friar in the Lombard region of Italy, delivered a sermon based on the game. ${ }^{\mathrm{I}}$

Les échecs sont l'image de la vie, c'est la raison pour laquelle Le Liber de moribus hominum et officiis nobilium ac popularium super ludo scacchorum contient, à cause de sa visée ouvertement didactique et moralisante, une série de modèles de conduite. Fondé sur un dispositif d'autorités issues de la littérature antique et de textes scripturaux, son ossature est solidement bâtie sur un réseau de récits exemplaires particulièrement persuasifs au sujet des dangers d'une longue liste de péchés, parmi lesquels les péchés capitaux ont un rôle de premier plan. Mais le traité propose également une riche casuistique des stratégies de construction d'un modèle idéal de société civile. Occulté par la fascination quasi hypnotique que la description des pions et de leurs mouvements sur l'échiquier exerce sur le lecteur,

I. The Book of Chess by Jacobus de Cessolis, translated \& edited by Hester L. Williams, New York, Italica Press, 2009, p. I. 
cet aspect est relativement peu étudié de nos jours. Il ne saurait être question, dans ces pages, de parcourir dans son intégralité l'histoire de la fortune inégale de ce bref traité qui a connu, entre le XIII ${ }^{\mathrm{e}}$ et le $\mathrm{XIV}^{\mathrm{e}}$ siècle, une diffusion hors du commun. Nous nous limiterons à rappeler brièvement les limites, aussi bien chronologiques que géographiques, de cette diffusion, extraordinaire et surprenante, ainsi que ses modalités.

Le Liber de Moribus est un bref traité rédigé en I275, en latin, par Jacques de Cessoles, dominicain originaire de Cessole, village de la région d'Asti ${ }^{2}$. Loin d'être un ouvrage technique consacré à l'exposition des règles d'un jeu pourtant très apprécié, au XIV $\mathrm{Xv}^{\mathrm{e}}$ siècle, aussi bien en milieu monastique que laïque, le Liber de Moribus utilise la structure formelle du jeu d'échecs pour présenter une société idéale dans laquelle chaque pièce ainsi que ses mouvements sur l'échiquier représentent le fonctionnement d'une cité idéale 3 . Beaucoup ont déploré le manque d'intérêt, à l'époque moderne, pour ce petit traité, quasiment oublié après le $\mathrm{XVI}^{\mathrm{e}}$ siècle, démontré, si besoin est, par l'absence d'éditions récentes des 250 à 300 manuscrits latins qui nous sont parvenus ${ }^{4}$. Mais avant d'être soudainement oublié, le Liber de Moribus fera l'objet, pendant deux siècles environ, d'une diffusion très importante, comme en témoigne la prolifération des copies latines ainsi que la multiplication de ses traductions en plusieurs langues : français ${ }^{5}$,

2. Les nombreuses copies manuscrites du Liber de Moribus livrent environ 3I formes différentes du nom de son auteur, parmi lesquelles figure également celle de Iacopo da Cessole. Cette onomastique floue, ajoutée à des données biographiques lacunaires, ont compliqué le travail de reconstitution de la biographie de Jacques de Cessoles. Après avoir fait l'objet d'un débat entre les partisans d'une origine picarde et ceux d'une origine lombardo-piémontaise, l'origine italienne de l'auteur a été établie assez tardivement : «[...] L'individu Jacques de Cessoles est vraisemblablement italien et certainement membre de l'ordre des Frères Prêcheurs. Cette appartenance est explicitée par la quasi-totalité des manuscrits conservés [...]. Une origine allemande ou française a parfois été évoquée à propos de Jacques, hypothèse qui ne repose sur aucune donnée précise.» Voir sur ce point Jacques de Cessoles. Le livre du jeu d'échecs ou la société idéale au Moyen Âge, XIIT siècle. Traduit et présenté par Jean-Michel Mehl, Paris, Stock-Moyen Âge, I995, p. 8. Sur l’origine italienne de Jacques de Cessoles voir également Th. Kaeppeli, "Pour la biographie de Jacques de Cessole», dans Archivium Fratrum Praedicatorum, 30, 1960, p. I49-162.

3. Bien que très brillamment utilisée dans l'ensemble du traité, la métaphore échiquéenne n'est pas une invention de Jacques de Cessoles; nous la devons à Jean de Galles, qui, entre I250 et I260 utilise, dans le Communiloquium sive summa collationum, les pièces du jeu d'échecs pour décrire la condition humaine. Il observe tout particulièrement qu’à la fin de la partie toutes les pièces, même les plus nobles, finissent, mélangées aux autres, au fond du même sac.

4. Voir sur ce point Jacques de Cessoles, Le Jeu des Eschaz moralisés, traduction de Jean Ferron, édition publiée par Alain Collet, Paris, Champion, 1999, ainsi que A. Scolari, «I volgarizzamenti del Libellus super ludus scaccorum. Prime indagini sulla tradizione", dans Studi di filologia italiana, ${ }^{\circ} 47$, Firenze, Accademia della Crusca, I989, p. 3I-99.

5. Trois traductions françaises du Liber de Moribus ont été réalisées entre 1335 et I347. Elles ont joué un rôle fondamental dans la diffusion de l'œuvre dans d'autres pays européens, car elles ont servi de support à d'autres traductions, notamment pour les traductions anglaises réalisées par l'éditeur-imprimeur William Caxton au $\mathrm{XV}^{\mathrm{e}}$ siècle. La première traduction française du traité est celle de Jehan de Vignay, réalisée entre 1335 et 1350 et intitulée Le Jeu des Eschés moralisés. Considérée comme la plus aboutie, le Jeu des Eschaz moralisés est la deu- 
allemand $^{6}$, anglais ${ }^{7}$, écossais ${ }^{8}$, toscan, catalan, néerlandais ${ }^{9}$, suédois et tchèque. Antonio Scolari justifie l'oubli dans lequel tombe le Liber de Moribus après le $\mathrm{XvI}^{\mathrm{e}}$ siècle par l'absence d'indications plus techniques sur les règles du jeu, qui n'intéressent pas réellement Cessoles :

[...] Il Libellus è la prima opera di un certo respiro dedicata al gioco degli scacchi che, penetrato in Europa forse dalla Spagna intorno all'anno 1ooo, conobbe subito una grande popolarità nella società medievale; l'opera non è però un manuale di gioco, e questo sarà uno dei motivi del repentino oblio in cui cadde nel XVI sec. allorché nacque la moderna letteratura scacchistica, ma è una lettura moralizzata della società umana assimilata alle pedine dello scacchiere ${ }^{10}$.

Il n'est assurément pas question, dans le cadre de cette étude, de nous lancer dans une analyse détaillée de chacune des traductions du traité. Il convient, en revanche, de souligner l'importance de ce travail. Chaque traduction comporte presque systématiquement une réécriture du texte latin, qui vise une adaptation au contexte social spécifique au pays auquel elle est destinée. Il s'agit là d'une entreprise dictée par la volonté, plus ou moins explicitement affichée en fonction des textes, de produire une représentation idéalisée des classes sociales spécifique au pays dans lequel la traduction est réalisée. C'est ainsi que les différents traducteurs modifient parfois en profondeur le texte de départ, tantôt en reconfigurant sa structure $^{\mathrm{II}}$ et en intégrant des récits exemplaires ou des références issues

xième traduction, réalisée par le dominicain Jean Ferron, et commencée le 4 mai 1347. La troisième traduction, vraisemblablement contemporaine de celles de Vignay et de Ferron, a été attribuée à un anonyme lorrain. Sur les traductions françaises du Liber de moribus, voir Jacques de Cessoles, Le Jeu des Eschaz moralisés, traduction de Jean Ferron, ouvr. cité, p. 9I.

6. Le Suisse Konrad von Ammenhausen, moine bénédictin né vers I300, achève autour de I337 le Schachzabelbuch, traduction allemande du Liber de moribus, à partir de l'original latin.

7. L'éditeur-imprimeur Anglais William Caxton édite deux fois, en I474 et en I483, sous le titre de The Game and Playe of Chesse une traduction qu'il avait lui-même réalisée à partir d'une compilation des versions françaises évoquées ci-dessus. Voir à cet égard R. H. Wilson, "Caxton's Chess Book", dans Modern Language Notes, $\mathrm{n}^{\circ}$ 62, 1947, p. 93-102.

8. En I5I5 John Asloan, notaire d'Édimbourg, écrit, en vers, une version écossaise du traité, le Buke of Chess. John Asloan ne cite pas explicitement Jacques de Cessoles comme source de son texte, toutefois clairement inspiré du Liber de Moribus.

9. Dat Scaecspel, réalisée autour de I400. Voir sur ce point J. Van Herwaarden «Dat Scaecspel: a profaneethical exploration", dans Journal of Medieval History, vol. 25, n 4, Abingdon, Taylor \& Francis, I999, p. 309-337.

Io. A. Scolari, «I volgarizzamenti del Libellus... », art. cité, p. 32.

II. S. Grossvogel, "Iacobus de Cessolis», dans Ch. Kleinhenz, Mediaeval Italy. An Encyclopedia, Londres, Routledge, 2004, vol. I, p. 573-574: "As Kaeppeli (1960) noted, the convent of San Domenico in Genoa produced a considerable amount of popular religious literature. It is not surprising therefore, that Ludus Scacchorum spread rapidly throughout the western and eastern Europe [...]. When Ludus Scacchorum was translated from latin into a vernacular, or from a vernacular into another, the content was sometimes modified to reflect a country's particular ways of representing its social classes." 
d'une culture inconnue à Jacques de Cessoles ${ }^{12}$, tantôt, au contraire, en retranchant des paragraphes entiers. Ces omissions sont justifiées soit par un souci d'économie soit par la nécessité d'effacer les références à la réalité trop spécifiquement lombardo-piémontaise sur laquelle s'appuient bon nombre d'exempla du Liber de Moribus ${ }^{13}$. Il n'en demeure pas moins que le programme développé par Cessoles dans son traité — proposer des modèles de comportement social adaptés à une société idéale - ne change pas de manière significative au gré des traductions. Notre étude a été réalisée à partir de la traduction toscane du Liber de Moribus, intitulée Volgarizzamento del libro dé costumi e degli offizii de’ nobili sopra il giuoco degli scacchi $i^{\mathrm{I}}$, rédigée dans la région de Florence par un auteur anonyme entre $\mathrm{I} 300$ et $133 \mathrm{O}^{\mathrm{Is}}$. La version toscane présente un intérêt majeur dans le cadre d'une étude sur le modèle au Moyen Âge. Les ajouts et les modifications qu'elle contient aboutissent, de manière bien plus marquée que dans l’original latin, à la création d'une modélisation pédagogique particulière-

I2. Hester L. Williams observe, à cet égard, que le souci d'adaptation à la réalité locale dont les traducteurs font preuve les amène également à enrichir le texte en augmentant ainsi sa longueur : "The [Book of Chess] was not just copied. It was translated, abbreviated, modified, turned into verse, and otherwise edited by substitutions and emendations that made material relevant to other regions and other cultures. [...] Subsequent emendations were comprised primarily of narratives and classical, biblical and legendary material. Some versions grew to more than four times the lenght of Cessolis' original." (The Book of Chess by Jacobus de Cessolis, translated and edited by Hester L. Williams, ouvr. cité, p. XIII.)

13. Il suffit de rappeler, sur ce point, l'exemple du travail de réecriture effectué par John Asloan dans le Buke of Chess, traduite en écossais et mise en vers : "It is clear [...] that not only has the author of Buke of Chess considerably shortened the latin original, in the first half of his poem, but that he has condensed [the text]. The condensation is effected in several ways, possibly with conscious planning to keep within a certain compass. [...] From the original text great quantities of quotations have been omitted, and similarly exempla are ignored for the sake of economy. " (The Buke of Chess, edited from the Asloan Manuscript (NLS MS I6500), edited by Catherine van Buuren, The Scottish Text Society, Edinburgh, 1997, p. CxxviI.)

I4. Nous nous appuierons plus précisément sur l'édition établie par P. Marocco, Volgarizzamento del Libro de' Costumi e degli offizii de' Nobili sopra il giuoco degli scacchi, tratto nuovamente da un codice Magliabechiano, Milano, Ferrario, I829. Pietro Marocco signale, dans l'introduction à son édition, l'existence de nombreux manuscrits toscans du Liber de Moribus, parmi lesquels une édition majeure, le Libro del giuocho di Scacchi intitolato de' costumi degl'huomini, et degli offitii de' nobili, Impresso in Firenze per Maestro Antonio Miscomini, I493, et une version plus médiocre, l'Opera nuova nella quale si insegna il vero reggimento delli huomini, e delle donne, ecc. composta per lo Reverendissimo Padre Frate Giacopo da Cesole sopra il giuoco delli scacchi, stampata in Vineggia per Francesco di Alessandro Bindoni, et Matheo Pasini compagni, I534.

I5. Pietro Marocco salue à ce propos l'excellent travail de traduction effectué par le traducteur toscan, au sujet duquel il ne peut toutefois pas fournir d'indications biographiques précises : "Per cui opera, ed in quale anno precisamente sia stato volgarizzato, né si sa, né sarebbe agevole a sapersi; certo è bene [...] apparir esso dettato nel secolo sopraddetto, ed anco nella sua prima metà. E ardisco soggiungere che fra le scritture di que' tempi questa traduzione va piuttosto colle piu apprezzevoli, che non colle mezzane; il che per avventura dié allo Zeno di asserire senz'altro argomento (ch'io mi sappia) esserne stato traduttore un Fiorentino o Toscano; dalla quale opinione io pure non vo lungi, considerata la somma purità delle voci, e quella spezialissima eleganza, e dilicatezza, che usiam chiamare atticismo, e che mai non si trova tanto, come nei trecentisti natii di quella provincia." (P. Marocco, Volgarizzamento del libro dé costumi..., ouvr. cité, p. v. Une datation plus précise [entre I300 et I330] a en revanche été établie par A. Scolari, "I volgarizzamenti del Libellus... », art. cité, p. 3I-99.) 
ment attentive à un projet de comportement idéal fondé sur l'harmonie sociale dans le domaine de la gestion de l'argent et de la propriété privée ${ }^{16}$.

D'un point de vue strictement formel l'échiquier idéal du Volgarizzamento ne diffère pas de celui évoqué par les versions latines et françaises. Composé de 64 cases noires et blanches, il comporte deux rangées de pièces par joueur. Chacune des pièces entretient des relations hiérarchiques et codifiées avec les autres pièces. C'est ce que rappelle Sandrine Thonon pour les échiquiers représentés dans les traductions françaises du Liber de Moribus :

La première ligne est occupée par les éléments nobles, actifs dans le royaume, et s'organise symétriquement autour du couple royal avec deux rochs, deux chevaliers, deux alphins ou juges. L'originalité et l'apport de l'auteur viennent au second rang, celui des pions dits "populaires». Ces pièces, au nombre de huit, n'ont généralement pas d'individualisation ni de qualification, mais Cessoles les investit d'un nom et d'une fonction afin de représenter l'ensemble de la société. [...] Chacun des pions entretient un des rapports privilégiés avec le pion noble situé au-dessus de lui. Plus le pion est proche du bord, plus il semble sortir des limites de la ville et moins il entre en contact avec le roi ${ }^{17}$.

On peut commenter brièvement, à la lumière, cette fois, du Volgarizzamento, les remarques proposées ci-dessus. Le traducteur anonyme reprend, sans la modifier, aussi bien l'individualisation que la qualification des pièces du jeu. Le couple royal est ainsi placé dans une position centrale, dans la rangée des pièces "nobles». Il est entouré des Alphins (Alfini), investis de la fonction de juges ${ }^{18}$, des Chevaliers, ainsi que de ses

16. Nous emploierons désormais l'abréviation Liber de Moribus pour évoquer le texte rédigé par Jacques de Cessoles. Nous ferons en revanche référence au Volgarizzamento del Libro dé Costumi lorsque nous parlerons de la version toscane anonyme. Il importe également de préciser que, dans le cas de l'anonyme toscan, il nous semble difficile de parler exclusivement de "traducteur", mais, en raison des écarts parfois très significatifs avec le texte du Liber de Moribus, plutôt de «rescripteur", voire même d' "auteur».

I7. S. Thonon, "Les métiers sur l'échiquier. Leurs représentation littéraire et figurée dans les traductions françaises de l'œuvre de Jacques de Cessoles ", dans M. Boone, E. Lecuppre-Desjardins, J. P. Sosson, Le verbe, l'image et la représentation de la société urbaine au Moyen Âge, Antwerpen-Apeldoorn, Garant, 2002, p. 2IO-2II.

18. L'auteur du Volgarizzamento fait preuve d'une grande précision dans la description des fonctions des Alphins, sans doute en raison de leur importance dans la relation que les différentes catégories socioprofessionnelles entretiennent dans le traité. Ainsi, le titre du chapitre qui leur est consacré, intitulé Della forma e dell'offizio degli Alfini, che sono giudici del reame, insiste sur leur rôle d'administrateurs de l'appareil judiciaire du royaume. Le début du chapitre se montre encore plus explicite sur ces points, en soulignant les différents domaines juridiques qui constituent le cadre de leur intervention : "Da sapere è che gli Alfini furono formati a modo di giudici assessori in cattedra col libro aperto dinanzi a gli occhi; e per alcuni piati sono di criminale, altri di possessione, e di quistioni di cose temporali, però fu bisogno, che dua giudici fussono nel reame, uno alfino nel bianco, e l'altro nel nero; e l'uno fosse a' piati civili, e l'altro a' piati criminali. L'offizio di coloro si è di consigliare il Re [...]. " (Volgarizzamento del libro de' costumi..., ouvr. cité, p. 26.) 
«suppléants» (vicarii), les Rochs (Rocchi) ${ }^{19}$. La rangée des pièces «populaires» est composée, quant à elle, du premier Pion, personnification des Laboureurs (Lavoratori della terra), et des Forgerons. Le troisième Pion symbolise plusieurs métiers : les Notaires, puis le Laneur (l'Arte della lana) et les Tanneurs (artefici di pelle e di coiame) ${ }^{20}$. Le quatrième Pion est en revanche associé au Marchands et aux Changeurs (Mercatanti e Cambiatori), le cinquième aux Médecins et aux Apothicaires (Medici di fisica e cirurgia e speziali). Viennent enfin les Taverniers, les Gardiens et officiers de la ville (septième Pion), puis les Messagers ${ }^{21}$. Soulignons d'emblée l'image sociale négative, ou du moins suspecte, des métiers représentés dans la rangée des pièces "populaires»; ainsi, le contact avec le sang ternit la réputation de médecins, chirurgiens, apothicaires, et ucciditori di animali; luxure et débauche guettent aubergistes, taverniers, ribauds et barattieri; enfin le maniement de l'argent frappe de discrédit marchands et changeurs ${ }^{22}$. Ce sont précisément ces métiers que Jacques de Cessoles s'efforce d'encadrer, dans le Liber de Moribus, par une structure déontologique et éthique stricte; cette démarche sera également retenue et développée par l'auteur du Volgarizzamento.

Le protocole de lecture du Volgarizzamento adopté dans les pages suivantes tient compte non seulement du symbolisme attribué à chacun des Pions, mais aussi du projet de société que sous-tend l'ensemble de l'œuvre.

19. "Vicarii ovvero legati del Re sono i Rocchi [...]. Però che il Re non può essere personalmente in ogni luogo del reame, si fece bisogno che l'autorità la quale è nel Re, si come nella fontana, discendesse a' suoi Rocchi, ovvero vicarii del Re; acciò ch'egli visitassono personalmente il reame, e manifestassono la potenzia del Re a tutti quegli che sono sottoposti alla signoria del Re." (Volgarizzamento de' costumi..., ouvr. cité, p. 48-49.)

20. En s'appuyant sur la description des outils associés à ces professions (la plume pour le notaire, les ciseaux pour le laneur et le couteau pour le tanneur), l'auteur entreprend d'expliquer les tâches confiées à ces catégories professionnelles : "Lo terzo popolare descriviamo in questo modo; [...] offizio di costoro è di recare in autentiche scritture le carte che si fanno per contratti, e di stare dinanzi a' giudici, leggere le condannagioni [...]. Gli altri hanno a tagliare panni, e cuscire, e tondere, e cignere, e tessere, e radere la barba, e cio sintende per le forvici. Per lo coltello, ovvero coltellaccio, sintendono calzolari, coiari d'ogni maniera, pellicciai, beccari, et ucciditori delli animali. Tutti questi s'appellano per nome di lanificio, però che tutti toccano pelli o pelo." (Volgarizzamento de' costumi..., ouvr. cité, p. 75-76.)

2I. Le huitième Pion est l'un des plus complexes de la rangée des pièces "populaires"; en plus des Messagers, il représente : "[...] gli scialacquatori e guastatori de' beni loro, [...] giocatori e puttanieri, [...] corrieri e portatori di lettere» (Volgarizzamento del libro de’ costumi..., ouvr. cité, p. II2-II3).

22. Voir sur ce point J. Le Goff, La bourse et la vie. Économie et religion au Moyen Âge, Paris, Hachette, I986, p. 50 : «Le Haut Moyen Âge avait condamné et méprisé beaucoup de métiers, d'abord interdits aux clercs, puis souvent aux laïcs, ou en tout cas dénoncés comme entraînant facilement au péché. Reviennent le plus souvent à l'index : aubergistes, bouchers, jongleurs, histrions, magiciens, alchimistes, médecins, chirurgiens, soldats, souteneurs, prostituées, notaires, marchands en première ligne, mais aussi foulons, tisserands, bourreliers, teinturiers, pâtissiers, cordonniers, jardiniers, peintres, prêcheurs, barbiers, baillis, gardes champêtres, douaniers, changeurs, tailleurs parfumeurs, tripiers, meuniers, etc." 
Selon Jenny Adams ${ }^{23}$, la moralisation des catégories professionnelles tentée par le Liber de Moribus serait particulièrement significative d'un glissement dans la conception de l'ordre social, jusque là représenté au moyen de la métaphore dite "organique" de l'État. Il s'agit là, on le sait, d'une représentation de l'ordre social inspirée par des textes scripturaires ainsi que par les réseaux métaphoriques employés par Jean de Salisbury dans le Policraticus, assimilant l'État au corps humain. Dans ce contexte, le roi représente la tête du corps social et ses sujets les différents membres, liés par conséquent entre eux par des liens organiques. Dans le Liber de Moribus, en revanche, la métaphore échiquéenne permet d'envisager une société où chaque catégorie professionnelle est à la fois individualisée et interdépendante des autres :

$[\ldots]$ the chess allegory imagines its subject to possess independent bodies in the form of pieces bound to the state by rules rather than biology. If the chess King advances, the pawns are not beholden to do the same. Nonetheless, this representation of subjects as independent of a central authority should not be mistaken for complete freedom ${ }^{24}$.

Sur ces bases, on peut s'interroger sur la nature des stratégies employées par l'auteur du Volgarizzamento pour aboutir à l'édification de ses lecteurs. Plus précisément, peut-on supposer l'existence, dans un contexte aussi explicitement social et politique, de modèles de comportement social vertueux communs à l'ensemble de la société? Les remarques générales présentées jusqu'ici laissent plutôt apparaître, dans le Volgarizzamento, l'existence de règles de comportement spécifiquement conçues pour chacune des catégories socio-professionnelles présentées dans le traité. Le procédé d'individualisation des pièces du jeu pourrait par conséquent nous permettre de répondre assez aisément à cette question : chaque pion symbolise un métier bien défini, et il est par conséquent évident de retrouver dans le texte des modèles éthiques spécifiques à chacune de ces catégories. Toutefois la question n'est pas si tranchée, en raison des nombreux parallèles que l'auteur du Volgarizzamento, tout comme Jacques de Cessoles, établissent entre des catégories sociales très différentes entre elles. C'est le cas du lien effectué entre les Chevaliers et le quatrième Pion "populaire», symbole des Marchands et des Changeurs. La nature de ce lien, essentiellement d'ordre financier, constitue un véritable point névralgique dans le traité : abondamment soulignée, elle est systématiquement liée

23. J. Adams, Power play. The Literature and Politics of Chess in the Late Middle Ages, Philadelphie, University of Pennsylvania Press, 2006, p. 29.

24. J. Adams, Power play..., ouvr. cité, p. 20. 
à l'autorité royale ${ }^{25}$. Nous allons donc adopter, dans les pages suivantes, une démarche comparatiste. Ainsi, notre étude s'appuiera-t-elle sur l'analyse des modèles que le Volgarizzamento réserve aux Chevaliers et aux Marchands-Changeurs. Dans un premier temps, nous proposerons, sur la base de ce corpus, une définition du cadre éthique et déontologique recevable dans le domaine militaire et commercial. Une deuxième partie sera consacrée à l'étude des modèles de maîtrise de l'harmonie sociale, pour la plupart liés à l'argent et à la propriété privée. Enfin, il conviendra d'analyser la stratégie de contrôle du modèle que l'auteur prévoit dans son traité.

Observons d'emblée que la création des modèles socio-professionnels du Volgarizzamento se conforme, dans chacun des chapitres du traité, à une sorte de canevas prédéterminé, qu'il s'agisse d'une pièce "noble» ou d'un Pion "populaire». Dans un premier temps une description physique de la pièce est proposée au lecteur, suivie de l'énumération des principes de base qui régissent sa fonction sociale. C'est ainsi qu'une panoplie guerrière présente aux lecteurs le Chevalier idéal :

Lo Cavaliere posto a cavallo, ornato di tutte le arme è formato in questo modo, chégli ebbe in capo l'elmo d'acciajo; la lancia in mano ritta; e lo scudo lo copria dalla mano manca; la spada e la mazza del ferro dalla detta mano, e'l coltello dalla mano ritta; la panziera in dosso; le corazze al petto; i gamberoli in gamba; i cosciali nelle coscie; li sproni in piede; et in ambe le mani i guanti del ferro; et il cavallo sotto savio et acconcio [...] tutto covertato ${ }^{26}$.

La description de l'armure du Chevalier, somme toute assez stéréotypée, n'est toutefois pas menée avec la même précision dans le cas des Marchands et des Changeurs, qui, en raison de leur profession moralement dangereuse, font l'objet d'une description plus fouillée que celle qui est réservée à la pièce "noble» :

Dinanzi del Re, devono sapere che sta il quarto popolare, il quale fue formato in forma d'uomo et avea nella mano ritta la bilancia col peso; nella mano manca avea la canna da misurare; alla cintola avea la borsa co' denari apparecchiata a rispondere a coloro che domandassono. Per costui sintendono mercatanti di panni e di tele, e di qualunque simigliante cosa, e ciò si dimostra nella canna da misurare; anche i cambiatori delle monete, e ciò si dimostra nella borsa ${ }^{27}$.

25. Voir sur ce point Volgarizzamento del libro dé costumi..., ouvr. cité, p. 85-86: "E convenevolmente si pongono dinanzi dal Re, però che debbono rispondere del soldo a' Cavalieri per lo Re."

26. Volgarizzamento del libro de' costumi..., ouvr. cité, p. 34-35.

27. Volgarizzamento del libro dé costumi..., ouvr. cité, p. 85. 
Le maniement de l'argent est le dénominateur commun de ces deux professions du commerce et du change, tout comme l'acceptation et la gestion des dépôts d'argent que les changeurs ajoutent à l'activité traditionnelle du change des devises. Si la balance désigne conjointement les activités du marchand et du changeur, la brasse, quant à elle, fait référence au commerce des tissus et des étoffes. La bourse, enfin, est liée aux activités du change. Observons, à cet égard, que l'auteur s'attarde sur les fonctions de cet élément, toujours à la disposition des clients (apparecchiata a rispondere a coloro che domandassono). De ce fait, elle ne répond pas à un usage personnel. Cet élément n'est pas sans rappeler le travail de réhabilitation des métiers du commerce entreprise dans la société médiévale et fondée sur l'argument de l'utilité commune de ces catégories professionnelles $^{28}$, ce que confirme la collocation du quatrième Pion sur l'échiquier : situé juste devant le Roi, il entretient un rapport privilégié avec l'autorité royale.

Conçue comme une liste chargée de susciter l'émulation tout en étant facilement mémorisable, l'énumération des principes fondateurs du modèle Chevalier et du Marchand idéal complète le portrait physique et est par la suite développée au moyen de récits exemplaires. Les qualités dont se compose le modèle idéal des professions du commerce concernent en premier lieu leur aptitude à gérer l'argent :

Tutti questi debbono fuggire avarizia; guardarsi da' debiti; debbono attendere et osservare la promessa e rendere interamente li depositi che sono loro accomandati ${ }^{29}$.

Un programme sévère est réservé à ces personnages, au sein duquel leur marge de manœuvre est fortement encadrée et balisée. Si les deux premiers points font référence au contact avec l'argent, les deux autres concernent en revanche l'usage de la parole dans un contexte professionnel; ces personnages sont par conséquent invités à respecter la parole donnée, et à rendre entièrement, sans recourir à des subterfuges, les dépôts d'argent qui leurs sont confiés lorsque leur propriétaire en demande la restitution ${ }^{30}$. Si

28. J. Le Goff, Marchands et banquiers au Moyen Âge, Paris, P.U.F., I956, p. 79 : «C'est [...] la notion d'utilité et de nécessité des marchands qui vint couronner l'évolution de la doctrine de l'Eglise et leur valut droit de cité définitif dans la société chrétienne médiévale. Très tôt, on mit en évidence l'utilité des marchands qui, en allant chercher au loin les marchandises nécessaires ou agréables, des denrées ou des objets introuvables en Occident et en les vendant dans les foires, fournissaient aux diverses classes de la société ce dont elles avaient besoin."

29. Volgarizzamento del libro dé costumi..., ouvr. cité, p. 85 .

30. Sur la relation entre les métiers du commerce et l'usage de la parole voir C. Casagrande et S. Vecchio, Les péchés de la langue. Discipline et éthique de la parole dans la culture médiévale, Paris, Cerf, I99I, p. 200. Les auteurs rappellent à cet égard l'association entre marchands et vice de la langue tel qu'il est évoqué dans de nombreux sermons dès le XII ${ }^{\mathrm{e}}$ siècle : "Ce sont plutôt certaines catégories professionnelles qui sont frappées 
la maîtrise de la parole et de l'argent est partie intégrante du cadre éthique sur lequel repose le modèle du Marchand et du Changeur, d'autres principes sont en revanche associés aux Chevaliers.

Le Chevalier est présenté comme un personnage hors concours, soumis à une discipline physique, morale et intellectuelle remarquablement exigeante :

Sapienza, fedeltà, larghezza, fortezza, misericordia, guardia dè pupilli, zelo delle leggi, tutte queste virtudi debbono essere nel cavaliere, chè come egli è armato d'arme corporale, così sia risplendente di costumi; però che quanto la dignità de' Cavalieri avanza gli altri di riverenza e d'onore, tanto più dee avanzare di virtudi. Con ciò sia cosa che onore non è altro che reddimento di reverenzia in testimonianza di virtudi. Savio dee essere il nobile cavaliere, et esperto, però che anzi che venga alla cintura cavalleresca, si conviene che sia usato d'armi; acciò ch'egli, ammaestrato per lunga esperienza sia acconcio a reggimento. Però che, essendo in alcuno modo la vita dé combattenti nelle mani del Cavaliere, laonde gli è commessa la cura della battaglia, si 'l conviene essere savio et assennato; imperò che più s'adopera nella battaglia l'arte e la sperienza del savio Cavaliere, che non fa la fortezza e l'ardire del non sperto. Bisogno è che quello popolo caggia in pericolo d'essere imprigionato, il cui Principe della cavalleria si sforza d'avere vittoria sopr'a nemici non per senno, ma per solo ardire. E però non chiami altri per capitani i giovani, però ch'egli è manifesta cosa ch'e' non sono assennati; ma chiaminsi uomini che sieno acconci ad essere fatti Cavalieri ${ }^{3}$.

Dans ces lignes, la supériorité intellectuelle du Chevalier est évoquée de manière récurrente; rappelons également le lien avec le pouvoir royal, souligné par l'allusion à la fidélité et au respect scrupuleux des lois (zelo delle leggi). Ces éléments s'accompagnent toutefois d'une véritable ritualisation du rôle du Chevalier. Afin d'obtenir le consensus et l'adhésion des lecteurs, sont intégrées dans leur portrait des conduites rituelles spécifiques, dont l'objectif est d'assurer la pérennité et l'efficacité d'un pouvoir et d'une société idéalisée ${ }^{32}$. Dans le cas des Chevaliers, l'auteur recourt à un rituel de passage, l'adoubement, doublé d'un rituel de type religieux (le baptême) dont le déroulement est évoqué dans les termes suivants :

E costoro quando si vengono a fare Cavalieri si bagnano il capo per significare, che debbono menare novella vita e novelli costumi. Vegghiano la notte in orazione, addomandando da Dio che doni loro di grazia quello che manca di natura. Per mano di Re

avec une virulence particulière. L'allusion récurrente aux marchands et aux avocats projette l'ombre sinistre d'un péché professionnel sur, précisément, les catégories professionnelles de la parole, dont la seule existence remet en cause le monopole sur le langage exercé depuis toujours par les ecclésiastiques. Les accuser systématiquement de mentir, c'est saper à la base la crédibilité et donc la prétendue autonomie de cette concurrence qui devient de jour en jour plus redoutable."

3I. Volgarizzamento del libro de' costumi..., ouvr. cité, p. 35.

32. Sur les relations entre rituels et société, voir Riti e rituali nelle società medievali, J. Chiffoleau, L. Martines et A. Parravicini Bagliani (éds), Spoleto, Centro di Studi sull'Alto Medioevo, 1994. 
o di Principe sono cinti, acciò che da lui abbiano la dignità e le spese di cui debbono essere guardiani ${ }^{33}$.

Les rituels chevaleresques évoqués dans ce chapitre ont une fonction précise : ils deviennent le miroir des classes sociales aristocratiques, dont ils ponctuent l'existence ${ }^{34}$. Le rituel de l'adoubement permet en effet, comme le souligne Stefano Gasparri, de rendre publique et explicite l'appartenance d'un individu à l'aristocratie militaire urbaine ${ }^{35}$. Ce cadre déontologique aboutit à la fixation du modèle social auquel toutes ces catégories professionnelles doivent se conformer et représente, de manière schématique, l'étendue et les limites de leurs fonctions.

Toutefois, l'auteur prend soin de proposer à ses lecteurs des stratégies de contrôle de l'ordre social : la maittrise de l'argent est, dans ce contexte, un instrument majeur. L'avarice fait par conséquent l'objet d'une analyse détaillée aussi bien dans le chapitre consacré aux Chevaliers que dans celui des Marchands et des Changeurs. L'évocation de ses conséquences néfastes occupe une bonne partie du chapitre consacré au quatrième Pion. Elle y est tout d'abord définie à l'aide d'une citation de Cicéron comme un amour excessif et désordonné des biens matériels, puis présentée comme un vice étendu à différentes catégories sociales, comme les Princes, des personnes âgées et des femmes :

Schifino adunque l'avarizia la quale è un servire agli idoli, della quale parla Tullio: Avarizia è cupidità di guadagno, o di retinere oltre che sia necessità, o vero è disordinato amore d'avere. Nullo vizio è peggiore di questo, massimamente ne' principi, et in coloro che governano la Repubblica. Questa è quella che sospinge l'uomo a qualunque male, e regna massimamente ne' vecchi; come questo addivenga, io non lo intendo. Qual cosa può essere più da non udire, che domandare per ispese nel viaggio più che non sia bisogno? Onde si legge ne' Proverbi de' Savi: l'avaro non fa veruna cosa dirittamente se non quando muore; al quale non pòi disiderare di peggio, ch'egli viva lungamente. L'avaro a nessun uomo è buono, a sé è rio, al povero è pessimo. Cagione di disdire non manca mai all'avaro. [...] L'avarizia della cupidezza è generamento di tutte le concupiscenze. [...] Egli è scritto nelli Proverbi de' Savi: alla pecunia si conviene comandare, non servire; se tu sai usare la pecunia ella è ancilla, se non sai, ella è donna; la pecunia non sazia l'avaro, ma accendegli più la sete e la fame. E Sallustio dice: l'avarizia pervertisce la fede e la probitade e l'altre buone arti, e per queste cose àe insegnato d'avere superbia e crudeltade, et avere per neente Domenedio, e tutte cose fa vendereccie ${ }^{36}$.

33. Volgarizzamento del libro de’ costumi..., ouvr. cité, p. 35.

34. Voir à cet égard Stefano Gasparri, «I rituali della cavalleria cittadina. Tradizioni militari e superiorità sociale nell'Italia del Duecento", dans J. Chiffoleau, L. Martines et A. Parravicini Bagliani, Riti e rituali..., ouvr. cité, p. 99.

35. S. Gasparri, art. cité, p. II2.

36. Volgarizzamento del libro de' costumi..., ouvr. cité, p. 88. 
L'insertion de ce passage dans un discours destiné à une catégorie professionnelle concernée par des contacts systématiques avec l'argent n'est pas une originalité en soi, puisque ces paragraphes sont en réalité la synthèse des éléments traditionnellement associés au péché d'avarice depuis les Pères de l'Église. Bien qu'attribuée ici à Cicéron, l'association de l'avarice à l'idolâtrie est également employée par saint Paul et associée résolument à l'adoration des richesses ${ }^{37}$. Ce motif est repris à la fin du passage, où, avec l'autorité de la citation attribuée à Salluste, l'auteur insiste sur le dévoiement que l'adoration illicite de l'argent constitue par rapport à celle, légitime, de Dieu (et avere per neente Domenedio). L'image de l'avare comme un être profondément anti-social est elle aussi topique :

Il vizio è per i laici tanto grave e pericoloso che lo è per gli uomini che appartengono all'istituzione ecclesiastica: come l'avarizia per monaci e chierici può arrivare a mettere in discussione la loro stessa identità di uomini di Dio e minare l'unità interna alla Chiesa, allo stesso modo l'avarizia dei laici mette in pericolo la loro appartenenza alla comunità sociale. L'avaro infatti non è solo ingiusto e dunque dannoso nei confronti dei suoi simili, egli è anche tendenzialmente un asociale tratto questo che gli era stato riconosciuto da sempre, sul quale molto avevano insistito prima i Padri, Basilio e Ambrogio, poi tutti gli altri. L'immagine è quella di un personaggio triste, solitario, abbandonato dagli amici, poco loquace, sempre sospettoso, spesso brusco e arrogante, nel migliore dei casi maleducato ${ }^{38}$.

Soulignons également, parmi les conséquences de l'avarice, la profonde frustration que ce vice engendre chez l'homme. Considérée, au même titre que l'Orgueil, la source de tous les maux, l'avarice est toujours responsable d'une insatisfaction perpétuelle, puisqu'elle ne parvient jamais à calmer la soif de richesses de l'avare. De même, s'il évoque l'amour des richesses qui le caractérise, en s'appuyant en cela sur la définition de Cassien, et le désir de retenir les richesses, l'auteur ne précise pas dans quelle mesure

37. Bien que cette définition de l'avarice apparaisse au moment où la circulation monétaire devient de plus en plus importante, elle est systématiquement employée lorsque ce n'est plus le cas. C'est plutôt en ces termes que C. Casagrande et S. Vecchio l'ont analysée : "Che cosè dunque l'avarizia? Idolatria, aveva scritto San Paolo in ben due occasioni (Ef. 5,5, Col. 3,5). [...] L'assimilazione all'idolatria è per l'avarizia una sorta di marchio di infamia che le resta impresso e che la segnala immediatamente come vizio di estrema gravità. [...] Fatto di metallo, che è materia densa e informe come il fango ma che ancora più del fango è vile perché è generato dalle viscere della terra, investito poi di una potenza che per sua natura non possiede, il denaro è - come l'idolo, e più di ogni altra forma di ricchezza, artificio, finzione, inganno. Tra i tanti dèi falsi ed ingannevoli che popolano l'affollato pantheon dell'avaro il dio denaro non è forse sempre il più importante, ma il più rappresentativo. Non stupisce dunque che la definizione dell'avarizia come amore del denaro sia quella che più si è imposta nel corso del tempo, anche in epoche di scarsa circolazione monetaria. [...] Ed è proprio come simbolo idolatrico che il denaro diventa il grande protagonista di una serie di scritti satirici, in prosa e in poesia, in latino e in volgare, la cui fortuna esplode alla fine del secolo XI per continuare poi nei secoli successivi.» (C. Casagrande et S. Vecchio, I sette vizi capitali. Storia dei peccati nel Medioevo, Turin, Einaudi, 2000, p. 103 et Io6.)

38. C. Casagrande et S. Vecchio, I sette vizi capitali..., ouvr. cité, p. I20. 
les biens accumulés dépassent des limites raisonnables ${ }^{39}$. En réalité, le Volgarizzamento accorde, à grand renfort d'exempla, une importance plus marquée à la description de l'avarice comme l'origine d'un bouleversement incontrôlable, incompatible avec tous les liens sociaux, dont elle provoque la destruction. La soif d'argent doit donc être savamment mầtrisée par les catégories professionnelles liées au maniement de l'argent. C'est ainsi que nous pouvons expliquer l'allusion, dans le portrait physique du Marchand qui nous est livré au début du chapitre, et notamment l'allusion à la bourse qu'il porte à la ceinture, toujours prête à répondre aux besoins de ses clients. Cette image fait référence à la circulation nécessaire des biens dans la société, seul remède contre la propagation de ce vice et seule condition nécessaire à une distribution équitable des richesses.

Dans le cas des Chevaliers le discours du Volgarizzamento établit une séparation entre cette catégorie sociale privilégiée et le maniement de l'argent. Les Chevaliers ne manipulent pas l'argent, et les allusions à des possessions personnelles sont soigneusement limitées. Ils reçoivent leur solde des Marchands, sur ordre du Roi, et sont invités à faire bon usage des biens mis à leur disposition :

Larghi conviene che siano gli Cavalieri, imperò che quando il Cavaliere ragguarda alla propia utilità, si prieme d'invidia di guadagno i suoi sottoposti e cavalcatori; ma quando veggiono ch'e' cavalcatori mettono le corpora loro a' pericoli, e gli loro segnori e guide intendono a multiplicare le spese e’ guadagni, piuttosto volgono le reni veggendo li nimici; et avviene spesse volte che colui che ha multiplicata la pecunia, non curando la vittoria, la tenacità dell'avarizia gli torna in ruina. A quanti sono oggi venute meno le potenzie, i quali stettero molto intesi e disiderarono d'avere abbondanza delle cose! Proveggia dunque il Cavaliere d'avere larghezza fra la gente sua, sì che l'avarizia nol tenti, e non creda che la tenacità sia guadagno, acciò che il nemico che l'ha in odio, fatto ricco dell'altrui avere, non solliciti il suo popolo da larghezza, acciò che quello che la natura della battaglia rifuta sanza forza s'abbia con la pecunia; però che spesse volte interviene, che quello che la natura niega, la pecunia vince. E però è da attendere con somma sollecitudine, che quando sopraviene pericolo di battaglia, ch'allora si faccia richiesta di pecunia ${ }^{40}$.

Le seul usage recevable de l'argent pour un Chevalier est le financement des guerres. En dehors ce contexte militaire, la soif d'argent, est,

39. Sur la définition de l'avarice par Cassien et la question d'une possession exagérée des richesses, voir R. Newhauser, The Early History of Greed. The Sin of Avarice in Early Mediaeval Thought and Literature, Cambridge, Cambridge University Press, 2000, p. 7-8: "If avarice was defined as the desire for more material wealth, the question still remained at what point "more" began. There was no authoritative answer to this problem. [...] Among the literary context [...] "more" did not come to imply the mere fact of private ownership." 40. Volgarizzamento del libro de’ costumi..., ouvr. cité, p. 4I. 
comme dans le cas des Marchands, envisagée comme un facteur de perturbation de l'équilibre social et militaire. Le refus de distribuer ou de faire circuler les richesses provoque inexorablement, au sein d'une armée, non seulement un autre péché capital, l'envie, mais aussi la trahison (piuttosto volgono le reni veggendo li nimici). Le contrôle de la cupidité impose également la maîtrise des biens demandés par ces personnages, qui ne se justifie qu'en cas de danger avéré (quando sopraviene pericolo di battaglia). À la lumière de ces considérations, nous pouvons conclure que, malgré des différences de statut social, le Volgarizzamento attribue une portée exemplaire à la gestion des richesses : ainsi, le "bon» marchand" ainsi que le «bon» Chevalier sont jugés sur le critère de l'investissement correct de l'argent.

Après avoir établi l'importance du critère financier dans les professions militaires et commerciales ainsi que son impact sur leurs modèles, il convient de nous interroger au sujet de l'efficacité des modèles édifiants proposés dans le texte. Bien évidemment, le recours aux exempla permet à lui seul, aussi bien dans le Liber de Moribus que dans le Volgarizzamento, de créer une série de modèles de conduite qui constituent un paradigme de vices et de vertus facilement mémorisable, faisant autorité et par conséquent infaillible ${ }^{4}$. Toutefois, Jenny Adams suggère l'existence, dans le Liber de Moribus, d'un double système de contrôle de ces modèles à la fois «externe» (par l'ensemble de la société, à savoir les lecteurs du livre) et «interne» (par les membres de la même catégorie professionnelle) :

Jacobus tries to solve this problem thus the structure of the game itself, which allows to him to use the power of shame and the threat of exposure to encourage a reader/a player to monitor his own actions. The stories in each chapter illustrate proper behavior via numerous exempla and sententiae. A Knight reading the Liber, for exemple, would see a model of his ideal behavior. Yet, at the same time, the public nature of this representations raises the possibility of shame and exposure ${ }^{42}$.

Force est de constater que la technique de l'exposition infamante au regard de la société est fréquemment employée, dans le texte, comme moyen de contrôle et de dissuasion. D’une manière générale, l’exposition, souvent outrageante, au regard de la société investit la structure même du

4I. Il convient de rappeler qu'aussi bien Cessoles que son traducteur toscan semblent se conformer, en tout et pour tout au précepte énoncé par Jean de Garlande à l'égard de l'exemplum (Exemplum est dictum vel factum alicuius autentice persone dignum imitatione). Toutefois, l'autorité des exemples choisis se fonde davantage, dans les textes latin et toscan, sur la source livresque des récits (auteurs de l'Antiquité classique ou textes scripturaux) que sur la notoriété des personnages qui en sont les protagonistes, à l'exception peut-être d'Oberto d'Asti, changeur à Gênes.

42. J. Adams, Power Play..., ouvr. cité, p. 35 . 
texte, par le biais de l'agencement des exempla à l'intérieur d'un même chapitre. Ainsi, un récit exemplaire construit autour d'un comportement recevable est brutalement juxtaposé à un exemple négatif, puis parfois à un exemple qui suscite une forte réaction émotive chez le lecteur. Les forfaits décrits dans les exempla sont ainsi d'abord mis en exergue, puis rehaussés au moyen de brèves formules qui font en même temps office de transition entre les différents récits. Dans le chapitre consacré aux Marchands et aux Changeurs, un meurtre épouvantable, commis par Settemulo par cupidité est commenté en ces termes : "Odiosa è questa avarizia di Settemulo, ma di quella di Tolomeo Re di Cipri è bene da ridere [... ${ }^{43}$ ". Aussi, les quatre principes fondateurs de l'identité sociale du quatrième Pion sont-ils rigoureusement disposés selon ce schéma. Les dangers de l'avarice sont exposés tout d'abord par un exemple de comportement recevable et vertueux, celui de Pauline, qui refuse une relation adultère malgré la promesse d'une importante somme d'argent :

Racconta Josefo nel decimottavo capitolo delle antichitadi, che fue una romana femmina la quale ebbe nome Paulina di chiara dignitade de' maggiori suoi, che intendea allo studio di castità, famosa d'openione d'onestade, ricca di molte ricchezze, bella della faccia, di quell'età che le donne si sogliono gloriare della castità. Questa fue maritata ad uno ch'avea nome Saturnino simigliante a lei di schiatta e di ricchezza, e degli altri ordinamenti. Dell'amore di lei era preso uno Cavaliere che avea nome Emerico Mondo, il quale sollevandola con doni e con promesse, neente poté ismovere l'animo della donna, duro come pietra, sì ch'ella volle anzi avere meno di quelle donora, che per avarizia perdere la castità del corpo ${ }^{44}$.

Dans cet exemple, la protagoniste jouit déjà d'un statut social privilégié - comme l'atteste non seulement la réputation de sa famille mais aussi celle qui lui est attribuée par l'opinion publique — et d'une fortune considérable, tout comme son époux. Le comportement correct vis-à-vis de l'argent consiste dans le refus de l'excès des richesses, qui permet également de préserver la chasteté. En revanche, l'exemple suivant concerne toujours un personnage féminin, illustration d'un rapport négatif à la richesse :

Leggiamo d'una femmina che menava vita solitaria, la quale poi che ebbe raunato una grande quantità d'oro, fece una fossa nel romitorio e nascoselvi entro: ma dopo la morte sua fatto assapere al Vescovo, per suo comandamento, l'oro fu gittato nel sepolcro suo, il quale oro poi che fu stato sotterra tre di, fu udita gridare: ohimé misera, che sono consumata di cotanta arsura. Quando ebbe data molta briga a' vicini per molte guise, per comandamento del Vescovo fue venuto a dissotterrarla, et aperto l'avello fue trovato

43. Volgarizzamento del libro de' costumi..., ouvr. cité, p. 88. Nous soulignons.

44. Volgarizzamento del libro de' costumi..., ouvr. cité, p. 86. 
che l'oro le era colato in bocca con fuoco di solfo; acciò che fosse avverato in lei quella parola che è scritta: d'oro avesti sete, d'oro beits.

L'aspect du cadavre après l'exhumation expose au regard de la société le péché commis par la femme avare ${ }^{46}$; observons également que le passage contient tous les éléments sociaux traditionnellement associés à l'avarice, notamment le comportement asocial de la femme avare (menava vita solitaria) et la tentative de préserver ses richesses après sa mort (fece una fossa nel romitorio e nascoselvi dentro ${ }^{47}$ ). Ce contre-exemple est suivi d'un troisième et d'un quatrième récit, présentés par l'auteur comme en mesure de susciter l'effroi et une violente réprobation pour le premier et le rire pour le second ${ }^{48}$.

Cette alternance, bien que moins approfondie que dans le chapitre sur le quatrième Pion, est également mise en ouvre dans le chapitre consacré aux Chevaliers. Un premier exemple célèbre en effet le rapport correct à l'argent dont fait preuve Alexandre le Grand, qui, au moyen de dons, parvient d'abord à gagner la fidélité des chevaliers du roi d'Inde, puis à s'emparer de l'Inde grâce à ces mêmes chevaliers ${ }^{49}$. Un deuxième exemple est en revanche conçu pour susciter l'émotion chez le lecteur car il relate le sacrifice héroïque du commandant de l'armée des Athéniens, qui accepte de mourir pour protéger ses chevaliers ${ }^{50}$.

Bien que l'agencement des récits exemplaires propose aux lecteurs un panel fortement persuasif, par leur juxtaposition, de modèles de comportement et des issues liées à chacun d'eux (exposition positive au regard de la société, ou bien infamante, ou encore source de dérision), le Volga-

45. Volgarizzamento del libro dé costumi..., ouvr. cité, p. 87.

46. Voir sur ce point J. Adams, Power Play..., ouvr. cité, p. 47.

47. Il s'agit là, bien évidemment, de la réactivation de l'image traditionnelle de l'avare, occupé à préserver ses richesses, très répandue dans les sermons depuis Cassien : «Secondo Cassiano esistono ancora popoli che la ignorano, e a suo parere [...] è sconosciuta nell'infanzia per poi dilagare nella vecchiaia. L'immagine topica del vecchio avaro, privo di forze e ormai prossimo alla morte, ma pur tuttavia ferocemente attaccato al suo tesoro, che ricorre più volte nei sermoni e negli exempla, si fonda su una convinzione ampiamente condivisa, di un legame privilegiato tra avarizia e vecchiaia [...].» (C. Casagrande et S. Vecchio, I sette vizi capitali..., ouvr. cité, p. II3.)

48. Volgarizzamento del libro de' costumi..., ouvr. cité, p. 88 : "[Tolomeo Re di Cipri] veggendo Antonio Imperadore, che quegli avea molte ricchezze et avendolo corretto di sozzura, veggendo che ne dovea perire, e per questo avendo messo nelle navi tutta la pecunia, et andato in alto mare acciò che forata la nave perisse a suo senno et Antonio nemico non avendo la preda, non sostenne di profondare l'oro e l'argento, ma rimandollo a casa per guiderdone della sua morte."

49. Volgarizzamento del libro de' costumi..., ouvr. cité, p. $43:$ : [...] i cavalieri di Porro sì l'abbandonaro, $e$ seguitando Alessandro come loro guida nelle castella, essendo onorati da lui di donamenti, vennero insieme col detto Alessandro contra il detto Porro re d'India, e si l'uccisero, e tutta l'India sottomisero alla signoria d'Alessandro."

5o. Volgarizzamento del libro de' costumi..., ouvr. cité, p. 44 : «Onde volle anzi morire perché vivessono i suoi, che vivere egli, vint' i suoi. Dolce cosa e bella si è morire per lo suo paese; nullo nomo sanza speranza d'avere grande beatitudine offrisce sé medesimo alla morte per lo paese." 
rizzamento met en place un dispositif encore plus impressionnant pour le lecteur, fondé sur le principe de la récompense. Observons, à cet égard, le résultat des actions entreprises par un Changeur et par un Marchand dans le chapitre consacré au quatrième Pion, puis celui obtenu par le duc de Turin dans le chapitre consacré aux Chevaliers.

Le Génois Oberto d'Asti, marchand-changeur, est victime d'un faux déposant, qui lui réclame la restitution d'un dépôt d'argent jamais effectué :

A Genova fue uno mercatante cambiatore, il quale ebbe nome Oberto, natio d'Asti; questi fue uomo di tanta lealtade che affermando alcuno falsamente d'aver fatto uno deposito appo lui di CC fiorini d'oro, et egli non trovando di ciò scritta veruna in sul libro della ragione, si come non dovea; né quello bugiardo inducendo sopra ciò testimonio alcuno, e'l mercatante lealissimo pur dicendo che quello deposito non avea ricevuto, vedendo il detto Oberto che quello rio uomo volle gridare, si 'l chiamoe incontanente e disse: taci figliuolo, e prendi CC fiorini d'oro, che tu di che diponesti appo me; et incontanente gli annoverò detta pecunia; si che volle anzi perdere la pecunia ingiustamente che sottrarre alcuna cosa alla sua buona fama ${ }^{\text {st }}$.

Bien que le faux déposant ne puisse produire aucune preuve de ce versement, ni écrite ni orale, il entreprend de diffamer le marchand, qui a bâti l'ensemble de son activité sur sa fiabilité. Oberto est confronté à un choix : il doit soit perdre de l'argent, soit s'exposer au risque de la perte de sa réputation professionnelle. Sans céder à l'avarice, il choisit de préserver son image sociale ${ }^{52}$. Toutefois, la validation de ce choix intervient immédiatement dans le texte, sous la forme d'une récompense pécuniaire, voulue par la volonté divine :

Il quale rio uomo, poi ch'ebbe avuta la detta pecunia in danno dell'anima sua, e, partito di quello mercatante, l'ebbe investita in mercatanzia, avvenne che per li tempi guadagnò $X V$ milia livre. Finalmente approssimandosi alla morte e non avendo figliuoli lasciò reda di tutto il suo, Oberto fedelissimo mercatante, affermando che de' CC fiorini d'oro ch'egli avea tolti fraudolentemente, aveva raunata cotanta pecunia; sicché

5I. Volgarizzamento del libro dé costumi..., ouvr. cité, p. 9I.

52. L'importance accordée à la réputation dans ce passage peut s'expliquer par la centralité des réseaux sociaux, indispensables, au Moyen Âge, aux activités du commerce et du change. Jean Favier observe, à cet égard, que celle-ci s’appuie précisément sur l'ancrage géographique ainsi que sur le relations sociales et familiales dans lesquelles cette catégorie professionnelle évolue et se développe : "L’origine géographique des citadins et les réseaux de solidarités familiales qui en découlent, déterminent à la fois les clientèles — fournisseurs et acheteurs - et les apports de main d'œuvre voire le recours aux intermédiaires. [...] Quand on sait que les associés sont individuellement responsables sur leurs patrimoines, on comprend qu'une fortune bien assise et bien maçonnée soit capable de rassurer les capitalistes sur ce qu'ils risquent en participant à la société, aussi bien de garantir les déposants contre le danger jamais oublié d'une banqueroute sans recours. Le gros investisseur comme le petit épargnant va regarder ce que vaut la garantie." (J. Favier, De l'or et des épices. La naissance de l'homme d'affaires au Moyen Âge, Paris, Hachette, 1987, p. 26 et 6o.) 
addivenne per disposizione della divina sentenzia, che questa cosa rimase ad Oberto fedelissimo mercatante ${ }^{53}$.

Cet exemplum soumet aux lecteurs un double avantage lié à un comportement socialement correct. Il est bien entendu efficace à long terme, car il permet d'éviter la damnation. Mais à court terme, il est sanctionné, assez rapidement, par l'obtention d'avantages matériels. Le principe inverse est appliqué au contre-exemple négatif qui suit. Dans ce cas, le comportement malhonnête d'un marchand est immédiatement réprimé par la perte des biens acquis par la fraude, qui sont restitués au client par une astuce particulièrement habile (une femme suggère au client malheureux de faire miroiter au marchand un dépôt d'argent bien plus important. Il s'agit bien entendu d'un faux trésor, caché dans des coffres bien fermés) :

Onde leggiamo che fue uno mercatante che fue molto famoso e di grandissima nominanza in guardare depositi, ma quando si vedea il bello tempo da disdire convenevole, sì era uno ladro. Udendo la fama di costui uno straniero, sì li lasciò in guardia un grande tesoro. In capo di tre anni, ritornò l'uomo al mercatante per richiederli il deposto tesoro appo lui, e 'l mercatante sappiendo che quegli non avea di ciò né carta né testimoni, negandoli il deposito disse che nol conoscea. Allora quegli, udendo questo, andavane piangendo e doloroso, e scontrandosi in una vecchiarella, questa li domandò la cagione di tanta tristizia; e quegli le rispuose: che hai tu a fare meco, buona donna? vatti per li fatti tuoi; e quella disse: io ti scongiuro che tu mi debbi dire perché tu vai così tristo; forse che ti potrò dare sano consiglio. Allora l'uomo, cosi vinto, le disse per ordine tutta la sciagura sua [...]. Andoe dunque l'uomo e fece secondo il detto della femmina, che, parlando gli amici suoi col mercatante del tesoro simulato che diceano di diporre appo lui, sopravvenne questi e richiese il deposito suo. Allora il mercatante disse: ben ti riconosco, e ben l'abbo guardato il deposito tuo; e disse al fattore suo: va dagliale. E così togliendosi l'uomo il deposito suo andonne allegro, e il mercatante non fedele, ingannato nella sua malizia, rimase con nulla ${ }^{54}$.

Dans ce récit, ce n'est pas la damnation de son âme qui est immédiatement promise au marchand voleur : c'est la dépossession des biens injustement acquis qui est obtenue par le biais des liens sociaux (les amis de la victime, qui l'aident à organiser le stratagème). Dans le chapitre des Chevaliers sont proposés d'autres exemples d'une récompense ou d'une sanction immédiate, envisageable à court terme, ayant la fonction de valider ou, au contraire de rejeter une conduite. Le récit exemplaire utilisé dans ce cadre est attribué par l'auteur à Paul Diacre, et concerne Onolfo, chevalier originaire de Parme, modèle de fidélité envers Perteric, son seigneur. Avec un stratagème Onolfo parvient à soustraire son seigneur à une

53. Volgarizzamento del libro de' costumi..., ouvr. cité, p. 90.

54. Volgarizzamento del libro de' costumi..., ouvr. cité, p. 9I. 
mort injuste, décidée par Grimoaldo, usurpateur du trône qui revenait de droit à Perteric, après l'assassinat de son frère, roi des Lombards, par Gariobaldo, duc de Turin. Capturé après avoir mis son seigneur en sécurité, Onolfo a finalement la vie sauve en raison de sa fidélité exemplaire. C'est en revanche le meurtrier du frère de Perteric, Gariobaldo de Turin, qui fait l'objet d'une punition exemplaire :

Quando fue fatto die, Onolfo e 'l fante suo furono presi dalla famiglia del Re Grimoaldo, et essendo isaminati del modo e della forma di come elli avevano liberato il loro signore Perteric, il re disse a' consiglieri suoi: che pena è da dare a costoro che hanno così fatto contra il volere e contra la maestade reale? Tra' quali consiglieri altri ve n'ebbe che disse, che tagliasse loro le teste; altri che gli facesse scorticare vivi; altri che gli ponesse in croce. Allora rispuose il Re e disse: Et io giuro per colui che mi fece venire in questo mondo, che costoro non sono degni di veruna morte, ma d'ogni onore; imperò che sono stati cosi fedeli al loro signore. Per la quale cosa il Re Grimoaldo sì diede loro altri donamenti, et il traditorie Gariobaldo Duca di Taurino fue miserabilmente morto a Taurino per mano del fante Godiberto di qua dietro Re; lo quale per suo tradimento avea privato della vita e del reame. E questo intervenne in una solenne festa, et entro la chiesa di San Giovanni Battistass.

La fidélité au seigneur, comportement prévu pour les Chevaliers, est dans ce cas récompensée par un renversement de situation; Onolfo a la vie sauve et est récompensé par le Roi ; en revanche, le duc de Turin, pourtant allié du Roi usurpateur et responsable de son accession au trône, connaît une mort infamante, comme le précise l'adverbe miserabilmente. Cet assassinat est d'ailleurs cautionné par la justice divine, comme réparation de la mort du roi légitime des Lombards : il se produit dans une église et est décrit comme une exécution publique, décidée par un décret de justice car il se déroule en présence de nombreux témoins (in una solenne festa).

Au terme de ce bref parcours, il convient de formuler quelques conclusions. Dans le domaine des professions liées au maniement de l'argent, pour des fins commerciales ou militaires, la structure conférée au texte par les exempla, permet, conformément aux suggestions envisagées pour le Liber de Moribus, de décrire aisément les stratégies employées par l'auteur du Volgarizzamento pour parvenir à l'édification de ses lecteurs. Celles-ci consistent en un paradigme précis de modèles de conduite fondés sur des attributions professionnelles strictement réglementées. D'un point de vue symbolique, ce programme s'appuie sur la description physique des différentes pièces du jeu, dont la panoplie symbolise à la fois l'étendue et les

55. Volgarizzamento del libro de' costumi..., ouvr. cité, p. 39. 
limites de leurs attributions au sein de la société; de ce point de vue, les modèles exposés par le texte s'appuient principalement, et traditionnellement, sur le principe de l'imitation. Les modèles éthiques et déontologiques prévus pour des classes sociales différenciées hiérarchiquement - différence symbolisée par leur position sur l'échiquier — se fondent sur des principes communs, notamment celui de la maitrise des ressources financières. Une différenciation s'effectue néanmoins par le recours à la ritualisation des fonctions des classes sociales privilégiées. Enfin, toujours dans une perspective d'ordre financier, ces modèles éthiques sont soumis à un dispositif de contrôle, réalisé au moyen de l'exposition au regard de la société ainsi que de celui de la sanction ou de la récompense matérielle immédiate, validant ou sanctionnant une conduite à court terme. Loin d'être le simple libretto écrit, d'après les dires de l'auteur, pour le divertissement des nobles, le Volgarizzamento est en réalité l'expression d'un projet bien plus structuré, celui de proposer un modèle de société fondé sur l'interdépendance financière des différentes catégories socio-professionnelles. 